\title{
In Situ Profiling of the Three Dominant Phyla Within the Human Gut Using TaqMan PCR for Pre-Hospital Diagnosis of Gut Dysbiosis
}

\author{
Young Jae Jo $^{1}$, Setu Bazie Tagele ${ }^{1}{ }^{\mathbb{D}}$, Huy Quang Pham ${ }^{1}$, YeonGyun Jung ${ }^{1}{ }^{\mathbb{D}}$, \\ Jerald Conrad Ibal ${ }^{1}$, SeungDae Choi ${ }^{1}$, Gi-Ung Kang ${ }^{1}$, Sowon Park ${ }^{2}$, Yunkoo Kang ${ }^{2}$, \\ Seung Kim ${ }^{2}$, Hong Koh ${ }^{2}$ and Jae-Ho Shin ${ }^{1, *(D)}$ \\ 1 School of Applied Biosciences, College of Agriculture and Life Sciences, Kyungpook National University, \\ Daegu 41566, Korea; dudwo7573@naver.com (Y.J.J.); setubazie@gmail.com (S.B.T.); \\ huypham@knu.ac.kr (H.Q.P.); jyg1076@knu.ac.kr (Y.J.); jerald.ibal@gmail.com (J.C.I.); \\ csd506@knu.ac.kr (S.C.); gukang@knu.ac.kr (G.-U.K.) \\ 2 Pediatric Gastroenterology, Hepatology and Nutrition, Severance Pediatric IBD Research Group, Severance \\ Children's Hospital, Yonsei University College of Medicine, 06229 Yonsei, Korea; sowon81@yuhs.ac (S.P.); \\ hollycow@yuhs.ac (Y.K.); pedks@yuhs.ac (S.K.); KHONG@yuhs.ac (H.K.) \\ * Correspondence: jhshin@knu.ac.kr; Tel.: +82-53-950-5716; Fax: +82-53-953-7233
}

Received: 2 February 2020; Accepted: 9 March 2020; Published: 11 March 2020

check for updates

\begin{abstract}
A microbial imbalance called dysbiosis leads to inflammatory bowel disease (IBD), which can include ulcerative colitis (UC). Fecal microbiota transplantation (FMT), a novel therapy, has recently been successful in treating gut dysbiosis in UC patients. For the FMT technique to be successful, the gut microbiota of both the healthy donors and UC patients must be characterized. For decades, next-generation sequencing (NGS) has been used to analyze gut microbiota. Despite the popularity of NGS, the cost and time constraints make it difficult to use in emergency services and activities related to the periodic monitoring of microbiota profile alterations. Hence, in this study, we developed a multiplex TaqMan qPCR assay (MTq-PCR) with novel probes to simultaneously determine the relative proportions of the three dominant microbial phyla in the human gut: Bacteroidetes, Firmicutes, and Proteobacteria. The relative proportions of the three phyla in fecal samples of either healthy volunteers or UC patients were similar when assessed NGS and the MTq-PCR. Thus, our MTq-PCR assay could be a practical microbiota profiling alternative for diagnosing and monitoring gut dysbiosis in UC patients during emergency situations, and it could have a role in screening stool from potential FMT donors.
\end{abstract}

Keywords: gut microbiota; next generation sequencing; primer design; qPCR; TaqMan probes; ulcerative colitis

\section{Introduction}

There are more than trillions of microorganisms inhabiting the human gut, and they live in homeostatic balance [1,2]. However, a microbial imbalance called dysbiosis causes metabolic disorders and affects the interactions of microorganisms in the host, which can eventually lead to inflammatory bowel disease (IBD) and ulcerative colitis (UC) [1,3]. The increasing global incidence of UC is growing, as is the concern for treating the disease $[4,5]$.

A novel therapy named fecal microbiota transplantation (FMT) has recently shown promise in treating gut dysbiosis in UC patients [6-8]. However, it is difficult to determine the safety and efficacy of FMT in UC patients, and it is also difficult to determine the factors that affect the success of FMT $[9,10]$. The success of FMT may be associated with microbial shift $[11,12]$. Previous studies have 
characterized gut dysbiosis as an alteration in the relative abundance of the two dominant phyla in the human gut, Bacteroidetes and Firmicutes [13]. Thus, for the FMT technique to be successful, it is crucial to characterize and monitor the gut microbiota of UC patients $[6,9]$.

For decades, next-generation sequencing (NGS) has been used to analyze changes in gut microbiota composition, and it has played a critical role in advancing gut microbiome research [14-16]. Despite the popularity of NGS, the cost and time constraints make it difficult to use for emergency services. A rapid, sensitive, and cost-effective method is required. Conventional and quantitative PCR methods have been useful to specifically detect organisms at the phylum level [17]. However, these methods are not able to simultaneously quantify different phyla in a single tube. TaqMan PCR is a powerful tool for the simultaneous detection and quantification of microbes in samples from different sources [18-20]. The relative composition of the three major phyla in the human gut, Bacteroidetes, Firmicutes, and Proteobacteria, has been proposed as a potential diagnostic tool for UC [9,21]. This study aimed to develop an MTq-PCR assay using novel TaqMan probes for profiling the dominant gut microbiota phyla. Our study confirmed that MTq-PCR is a viable alternative to NGS when characterizing the three dominant phyla (Bacteroidetes, Firmicutes, and Proteobacteria) in fecal samples from healthy and UC patients.

\section{Results}

\subsection{Specificity of TaqMan Probes and Primers}

In this study, phylum-specific probes (Table 1) were developed, and their phylum specificity was evaluated in silico using the SILVA database (Quast et al., 2013). The Bacteroidetes TaqMan probe (Bat1) matched $93 \%$ of the $16 \mathrm{~S}$ rRNA genes that were classified under Bacteroidetes in the SILVA database when two mismatches per probe were allowed (Table 2). The Bat1 probe had low sensitivity $(3.7 \%)$ to non-target $16 \mathrm{~S}$ rRNA gene sequences classified under Firmicutes (Table 2). Interestingly, the Bat1 probe had no predicted specificity to Enterobacteriaceae, which are the gut-dominant Proteobacteria. The previous CFB555f and 798cfbF probes covered a large percentage ( 96.7 and $98.2 \%$, respectively) of Bacteroidetes target sequences; however, both showed very weak mismatching capabilities against non-targets such as Firmicutes and Proteobacteria (Tables 3 and 6).

The Firmicutes probe (Fir7) covered 55.7\% of its intended phyla but had very low coverage (less than $4 \%$ ) for the non-targeted phyla (Table 2). Previously reported Firmicutes-specific probes had high $(>96 \%)$ coverage for Firmicutes target sequences; however, they showed very weak mismatching capabilities against non-target gene sequences from Bacteroidetes and Proteobacteria (Table 4 and Table 6). Interestingly, the Proteobacteria probe (Pro3) was not predicted to bind to non-targeted phyla. Pro3 was highly specific (94\%) to Enterobacteriaceae (Table 2). Although the Pro3 probe had lower Proteobacteria coverage than the previous Proteobacteria-specific probe, our probe had comparable efficacy in terms of gut-dominant Proteobacteria (Enterobacteriaceae). More importantly, our probe showed strong mismatching capabilities against non-targeted genes from Firmicutes and Bacteroidetes (Tables 5 and 6). Overall, our probes had very low coverage (less than $4 \%$ ) to non-targeted taxa (Table 2). The three probes were designed to bind within the 16S rRNA region of the forward (Eub268) and reverse (Eub797) primers without overlapping (Figure 6). 
Table 1. Probe and primer design.

\begin{tabular}{|c|c|c|c|c|c|c|}
\hline Oligonucleotide & Primer/Probe Name & Target Phylum & Fluoro-phore $^{\text {a }}$ & Quencher & Sequence $\left(5^{\prime}-3^{\prime}\right)$ & References \\
\hline \multirow[t]{4}{*}{ Probe } & Bat1 & Bacteroidetes & 6-FAM & BHQ-1 & GAGGCAGCAGTGAGGAATATTGGT & This study \\
\hline & Fir7 & Firmicutes & HEX & BHQ-1 & AAGGCGACGATCGGTAGCCGRM * & This study \\
\hline & Pro3 & Proteobacteria & Cy5 & BHQ-2 & GCCTTCGGGTTGTAAAGTACTTTCAGC & This study \\
\hline & Eub518 & Eubacteria & TEX615 & BHQ-2 & ATTACCGCGGCTGCTGG & [22] \\
\hline Forward primer & Eub268 & Eubacteria & & & TWGGYGRGGTAACGGCYCACCWA & This study \\
\hline Reverse primer & Eub797 & Eubacteria & & & GGACTACCAGGGTATCTAATCCTGTT & [23] \\
\hline
\end{tabular}

a Fluorophores were coupled to the most effective probe candidates. ${ }^{*} \mathrm{RM}$ is an ambiguous nucleotide code where $\mathrm{R}$ stands for $\mathrm{A}$ or $\mathrm{G}$, and $\mathrm{M}$ stands for $\mathrm{A}$ or $\mathrm{C}$.

Table 2. In silico PCR assay with newly designed probes.

\begin{tabular}{ccccc}
\hline Probe & Target Taxon & \multicolumn{2}{c}{ Percent Coverage in the Target Taxon (\%) ${ }^{\mathbf{a}}$} \\
\hline \multirow{2}{*}{ Bat1 } & & Perfect Match & 1 Mismatch & 2 Mismatches \\
\hline & Bacteroidetes & 63.7 & 85.2 & 93.0 \\
\hline Firmicutes & 0.0 & 0.1 & 3.7 \\
\hline & Proteobacteria & 0.0 & 0.2 & 39.4 \\
\hline & Enterobacteriaceae & 0.0 & 0.0 & 0.0 \\
\hline & Firmicutes & 2.1 & 13.5 & 55.7 \\
\hline Pro3 & Bacteroidetes & 0.0 & 0.0 & 0.6 \\
\hline & Proteobacteria & 0.0 & 0.1 & 0.1 \\
\hline & Enterobacteriaceae & 0.0 & 0.0 & 21.7 \\
\hline & Proteobacteria & 7.1 & 13.6 & 94.0 \\
\hline & Enterobacteriaceae & 75.0 & 92.1 & 0.0 \\
\hline
\end{tabular}

a Percent sequence coverage in the target group based on the online "TestProbe" tool in the SILVA 138 SSU Ref NR database, released in January 2020. 
Table 3. Comparison of our probe with previously reported Bacteroidetes-specific probes.

\begin{tabular}{|c|c|c|c|c|c|c|c|}
\hline \multirow[t]{2}{*}{ Probe } & \multirow[t]{2}{*}{ Sequence $\left(5^{\prime}-3^{\prime}\right)$} & \multirow[t]{2}{*}{ Location * } & \multicolumn{2}{|c|}{ Gut Bacteroidetes (\%) ${ }^{\text {a }}$} & \multirow[t]{2}{*}{ All Bacteroidetes (\%) ${ }^{a}$} & \multirow[t]{2}{*}{ All Other Bacteria (\%) ${ }^{a}$} & \multirow[t]{2}{*}{ Reference } \\
\hline & & & Bacteroides spp. & Provotella spp. & & & \\
\hline Bat1 & GAGGCAGCAGTGAGGAATATTGGT & $346-369$ & 98.5 & 97.9 & 86.2 & 28.0 & This study \\
\hline S-P-Bdet-0107-a-S-21 & GCACGGGTGMGTAACRCGTAT & $107-127$ & 91.9 & 95.9 & 78.7 & 23.7 & [24] \\
\hline CFB555f & CCGGAWTYATTGGGTTTAAAGGG & 555-577 & 96.4 & 97.8 & 96.7 & 25.3 & [25] \\
\hline $798 \mathrm{cfbF}$ & CRAACAGGATTAGATACCCT & 779-798 & 97.8 & 98.5 & 98.2 & 25.3 & [26] \\
\hline
\end{tabular}

"Escherichia coli position. a Percent sequence coverage in the target group based on the online "TestProbe" tool in the SILVA 138 SSU Ref NR database, released in January 2020.

Table 4. Comparison of probes from the current study with previously reported Firmicutes-specific probes.

\begin{tabular}{|c|c|c|c|c|c|c|c|}
\hline \multirow[t]{2}{*}{ Probe } & \multirow[t]{2}{*}{ Sequence $\left(5^{\prime}-3^{\prime}\right)$} & \multirow[t]{2}{*}{ Location * } & \multicolumn{2}{|c|}{ Gut Firmicutes (\%) ${ }^{a}$} & \multirow[t]{2}{*}{ All Firmicutes $(\%)^{a}$} & \multirow[t]{2}{*}{ All Other Bacteria $(\%)^{\text {a }}$} & \multirow[t]{2}{*}{ Reference } \\
\hline & & & Clostridiaceae & Eubacteriaceae & & & \\
\hline Fir7 & AAGGCGACGATCGGTAGCCGRM & $272-290$ & 75.6 & 8.5 & 55.7 & 24.1 & This study \\
\hline S-P-Firm-0352-a-S-18 & CAGCAGTAGGGAATCTTC & $352-369$ & 1.4 & 0.6 & 46.9 & 24.2 & [24] \\
\hline S-P-Firm-0525-a-A-18 & ACCTACGTATTACCGCGG & $525-542$ & 98.2 & 95.5 & 96.6 & 80.1 & [24] \\
\hline Firm350f & GGCAGCAGTRGGGAATCTTC & $350-369$ & 95.7 & 96.0 & 87.3 & 72.2 & [25] \\
\hline 928F-Firm & TGAAACTYAAAGGAATTGACG & $905-925$ & 94.2 & 93.8 & 96.2 & 96.4 & [26] \\
\hline
\end{tabular}

* Escherichia coli position. a Percent sequence coverage in the target group based on the online "TestProbe" tool in the SILVA 138 SSU Ref NR database, released in January 2020.

Table 5. Comparison of probes from the current study with previously reported Proteobacteria-specific probes.

\begin{tabular}{|c|c|c|c|c|c|c|}
\hline Probe & Sequence $\left(5^{\prime}-3^{\prime}\right)$ & Location * & Gut Proteobacteria (Enterobacteriaceae) (\%) ${ }^{a}$ & All Proteobacteria $(\%)^{\text {a }}$ & All other Bacteria $(\%)^{\text {a }}$ & Reference \\
\hline Pro3 & GCCTTCATACCACGGAGTACTTTCAGC & $415-441$ & 94.0 & 21.7 & 6.3 & This study \\
\hline Gamma395f & CMATGCCGCGTGTGTGAA & $395-412$ & 96.3 & 85.6 & 31.7 & [25] \\
\hline Gamma877F & GCTAACGCATTAAGTRYCCCG & $859-880$ & 0.3 & 9.3 & 31.8 & [27] \\
\hline
\end{tabular}

* Escherichia coli position. a Percent sequence coverage in the target group based on the online "TestProbe" tool in the SILVA 138 SSU Ref NR database, released in January 2020. 
Table 6. Comparison of the specificity between probes from the current study with those previously reported.

\begin{tabular}{|c|c|c|c|}
\hline Target Phylum & Probe/Primer & Target Taxon & Percent Coverage in the Target Taxonomy (\%) \\
\hline \multirow[t]{16}{*}{ Bacteroidetes } & Bat1 * & Bacteroidetes & 86.2 \\
\hline & & Firmicutes & 3.7 \\
\hline & & Proteobacteria & 39.4 \\
\hline & & Enterobacteriaceae & 0.0 \\
\hline & S-P-Bdet-0107-a-S-21 & Bacteroidetes & 78.7 \\
\hline & & Firmicutes & 13.9 \\
\hline & & Proteobacteria & 13.1 \\
\hline & & Enterobacteriaceae & 0.2 \\
\hline & CFB555f & Bacteroidetes & 96.7 \\
\hline & & Firmicutes & 45.3 \\
\hline & & Proteobacteria & 0 \\
\hline & & Enterobacteriaceae & 0 \\
\hline & & Bacteroidetes & 99.4 \\
\hline & $798 \mathrm{cfbF}$ & Firmicutes & 99.0 \\
\hline & & Proteobacteria & 99.2 \\
\hline & & Enterobacteriaceae & 99.4 \\
\hline \multirow[t]{20}{*}{ Firmicutes } & Fir7* & Firmicutes & 55.7 \\
\hline & & Bacteroidetes & 0.6 \\
\hline & & Proteobacteria & 3.9 \\
\hline & & Enterobacteriaceae & 0.1 \\
\hline & S-P-Firm-0525-a-A-18 & Firmicutes & 96.6 \\
\hline & & Bacteroidetes & 82.2 \\
\hline & & Proteobacteria & 80.2 \\
\hline & & Enterobacteriaceae & 93.2 \\
\hline & S-P-Firm-0525-a-S-18 & Firmicutes & 96.6 \\
\hline & & Bacteroidetes & 82.2 \\
\hline & & Proteobacteria & 80.2 \\
\hline & & Enterobacteriaceae & 93.2 \\
\hline & Firm350f & Firmicutes & 87.3 \\
\hline & & Bacteroidetes & 14.3 \\
\hline & & Proteobacteria & 93.7 \\
\hline & & Enterobacteriaceae & 92.9 \\
\hline & 928F-firm & Firmicutes & 96.2 \\
\hline & & Bacteroidetes & 97.5 \\
\hline & & Proteobacteria & 95.6 \\
\hline & & Enterobacteriaceae & 92.8 \\
\hline \multirow[t]{12}{*}{ Proteobacteria } & Pro3* & Proteobacteria & 21.7 \\
\hline & & Enterobacteriaceae & 94.0 \\
\hline & & Bacteroidetes & 0.0 \\
\hline & & Firmicutes & 0.0 \\
\hline & Gamma395f & Proteobacteria & 85.6 \\
\hline & & Enterobacteriaceae & 96.3 \\
\hline & & Bacteroidetes & 2.3 \\
\hline & & Firmicutes & 21.0 \\
\hline & Gamma877F & Proteobacteria & 9.3 \\
\hline & & Enterobacteriaceae & 0.3 \\
\hline & & Bacteroidetes & 1.2 \\
\hline & & Firmicutes & 33.4 \\
\hline
\end{tabular}

* Probes designed in this study are indicated in bold. 


\subsection{In Vitro Evaluation of the Multiplex Taqman Assay}

The specificity of the probes used to detect three bacteria strains (Bacteroides fragilis GUT-04, Clostridium butyricum TO-A, and Shigella sonnei KCCM 41282) within different phyla was evaluated in a singleplex and multiplex conventional PCR reaction. In the singleplex PCR assay, primer pairs for Eubacteria, Eub268, and Eub797 successfully amplified a single band of 529 bp from each of the three bacterial strains (Figure 1A-C). However, the probe designed for Bacteroidetes, Bat1, only amplified the target strain, B. fragilis, and no PCR product was detected for non-target strains. Similarly, the Fir7 and Pro3 primers only amplified their intended target strain (Figure 1B and C), suggesting that the probes were specific. In the multiplex PCR, wherein a mock community of three strains was constructed, the probes specifically amplified their target strains, and the probes were sensitive enough to discriminate all strains mixed in a single tube (Figure 2). These results indicated that the designed primer and probe sets did not interfere with each other in the multiplex PCR reaction. In contrast, primers designed to detect Eubacteria failed to discriminate between the strains used in the PCR reaction (Figure 2).

A

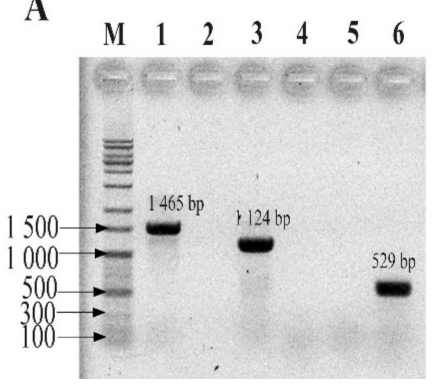

B

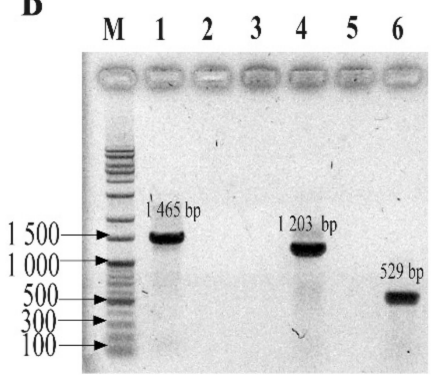

$\mathrm{C}$

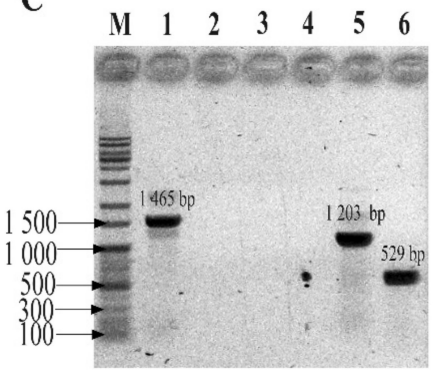

Figure 1. Agarose gel electrophoresis of singleplex PCR products produced by phylum-specific probes for (A) Bacteroides fragilis GUT-04, (B) Clostridium butyricum TO-A, and (C) Shigella sonnei KCCM 41282. M: marker 1kb DNA ladder; Lane 1: 27F/1492R; Lane 2: negative control; Lane 3: Bat1/1492R; Lane 4: Fir7/1492R; Lane 5: Pro3/1492R; and Lane 6: Eub268/Eub797. The universal primer set, 27F/1492R, without template DNA, served as the negative control.

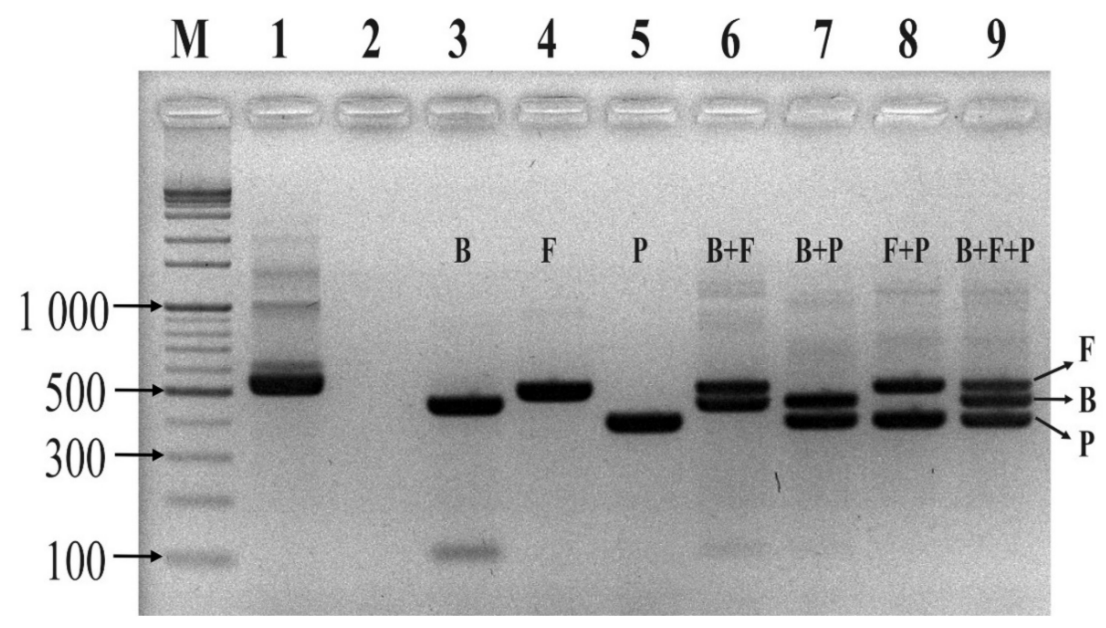

Figure 2. Agarose gel electrophoresis of multiplex PCR products. Letters B, F, and P signify the DNA template extracted from B. fragilis GUT-04, C. butyricum TO-A, and S. sonnei KCCM 41282, respectively. $\mathrm{B}+\mathrm{F}, \mathrm{B}+\mathrm{P}, \mathrm{F}+\mathrm{P}$, and $\mathrm{B}+\mathrm{F}+\mathrm{P}$ represent mock communities. M: marker 1kb DNA ladder; Lane 1: positive control; Lane 2: negative control; Lane 3: Bat1+Eub797; Lane 4: Fir7+Eub797; Lane 5: Pro3+Eub797; Lane 6: Bat1+Fir7+Eub797; Lane 7: Bat1+Pro3+Eub797; Lane 8: Fir7+ Pro3+Eub797; and Lane 9: Bat1+Fir7+Pro3+Eub797. A eubacterial primer set, Eub268/Eub797, with template DNA from the mock community without probes served as the positive control, and a primer set, 27F/1492R, without template DNA, served as the negative control. 


\subsection{Comparison of Multiplex TaqMan $q P C R(M T q-P C R)$ and NGS}

The sensitivity of the MTq-PCR and NGS methods in determining the relative proportions of the three phyla from fecal samples collected from healthy and UC patients were compared. The relative proportions of the three phyla in fecal samples of either healthy volunteers or UC patients were similar for both methods. In fecal samples from healthy individuals, the relative proportions of Bacteroidetes and Firmicutes were much higher than the proportion of Proteobacteria. In contrast, in UC patients, both methods showed that the percentage of Bacteroidetes was very low. In both methods, the Firmicutes/Bacteroidetes ratio was close to 0.5 in fecal samples of healthy individuals, but the ratio was very high in UC patients. More importantly, both methods revealed that the proportion of Proteobacteria was high in UC fecal samples when compared to healthy individuals (Figures 3 and 4).

A) NGS

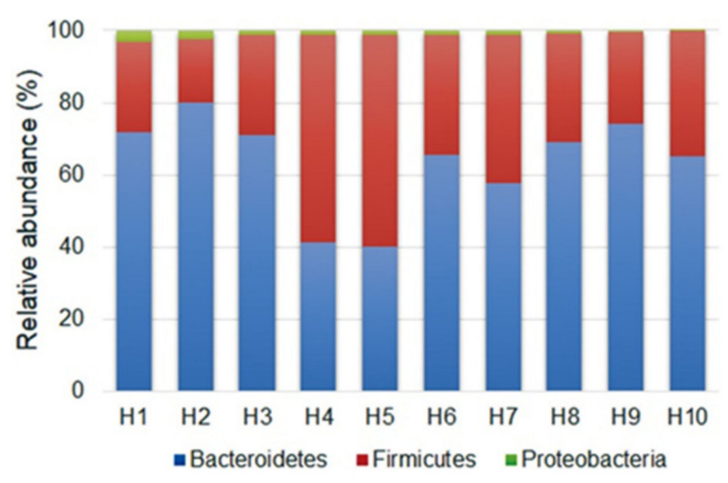

B) $\mathrm{MTq}-\mathrm{PCR}$

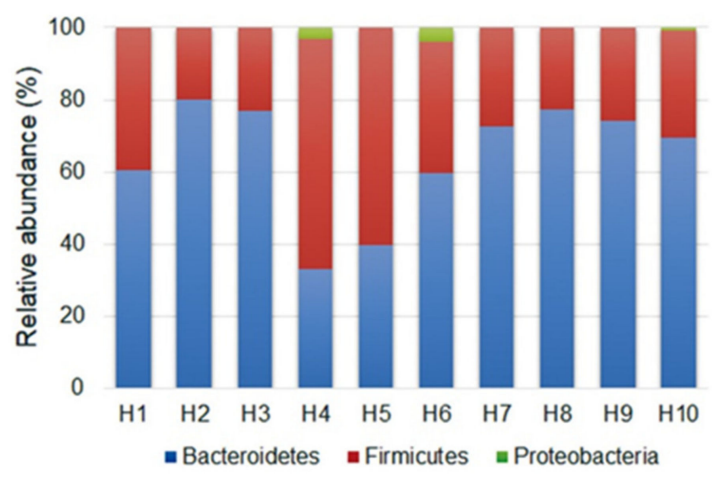

Figure 3. Relative proportions of the three phyla from fecal samples of healthy subjects determined by (A) NGS and (B) MTq-PCR.

\section{A) NGS}

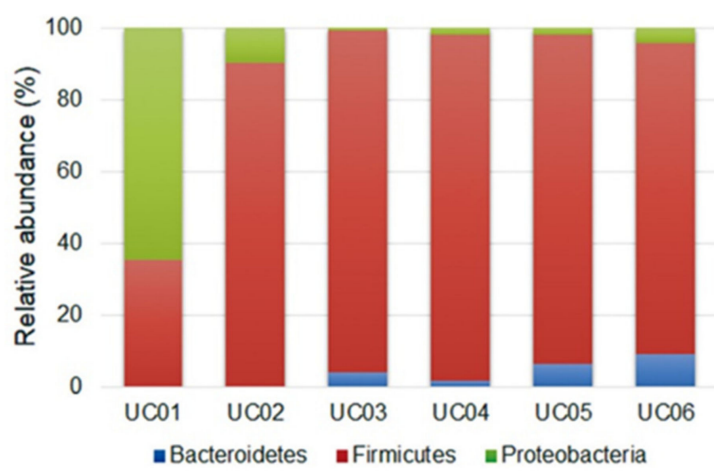

\section{B) MTq-PCR}

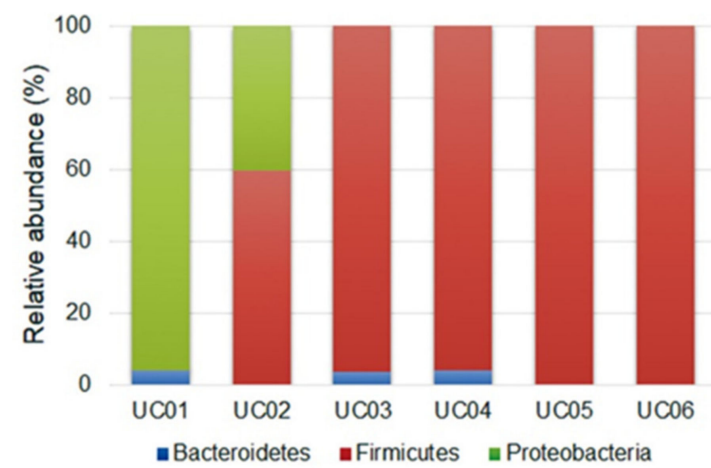

Figure 4. Relative proportions of the three phyla from fecal samples of UC patients determined by (A) NGS and (B) MTq-PCR.

Principal coordinate analysis (PCoA) was performed to compare the profiling efficiency of NGS and MTq-PCR (Figure 5). The relative proportions of the three dominant phyla in UC patients and healthy volunteers were used for the analysis. The clustering of UC patients and healthy volunteers using NGS was similar to the clustering seen when using MTq-PCR, which indicated the potential of MTq-PCR for monitoring microbiota profile alterations.

Furthermore, the statistical analysis examining the similarity between NGS and MTq-PCR was analyzed using multivariate similarity Anosim and Adonis tests with the Bray-Curtis distance. Based on the Anosim and Adonis analysis, NGS and MTq-PCR were not significantly different $(p>0.05)$ in profiling the abundance of the three phyla from fecal samples of either healthy volunteers or UC 
patients (Table 7). Both NGS and MTq-PCR were able to significantly discriminate $(p<0.001)$ the phylum composition between healthy volunteers and UC patients (Table 8).

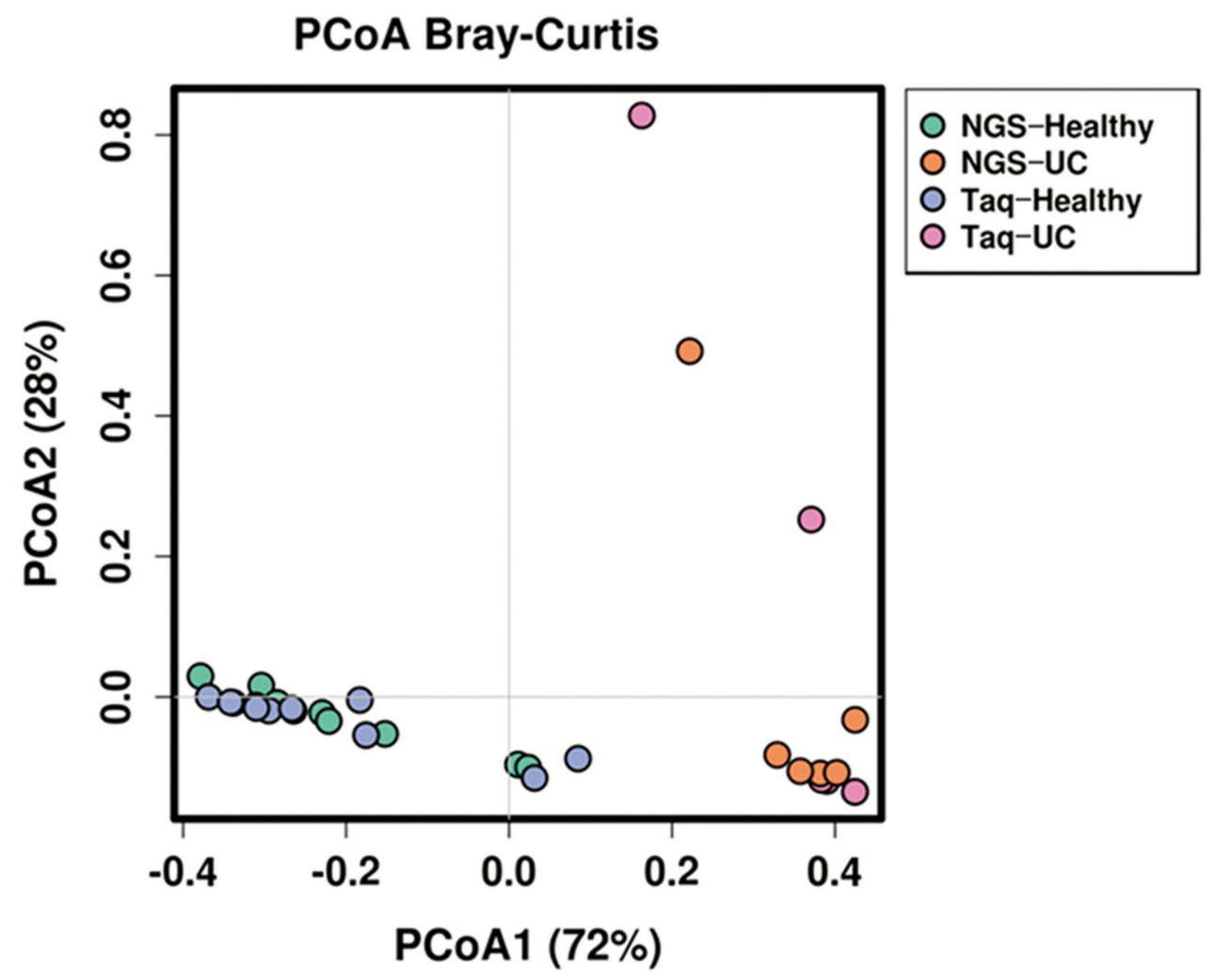

Figure 5. Principal coordinate analysis of (PCoA) of the three dominant phyla in the fecal samples of UC patients and healthy volunteers using NGS and MTq-PCR.

Table 7. Adonis and Anosim statistical analyses between NGS and MTq-PCR using fecal samples collected from healthy subjects and UC patients.

\begin{tabular}{clcccc}
\hline Sample Source & Comparison & \multicolumn{2}{c}{ Adonis $^{\mathbf{a}}$} & \multicolumn{2}{c}{ Anosim $^{\mathbf{a}}$} \\
\hline & & $\mathbf{R}^{\mathbf{2}}$ & $\boldsymbol{p}$ Value & $\mathbf{R}$ & $\boldsymbol{p}$ Value \\
\hline Healthy & NGS vs. MTq-PCR & 0.002 & 0.868 & -0.049 & 0.779 \\
UC & NGS vs. MTq-PCR & 0.017 & 0.73 & 0.019 & 0.328 \\
Across all samples & NGS vs. MTq-PCR & 0.001 & 0.961 & -0.04 & 0.89 \\
\hline
\end{tabular}

${ }^{a}$ Adonis and Anosim statistical analyses were carried out based on Bray-Curtis dissimilarities at the phylum level.

Table 8. Adonis and Anosim statistical analyses between NGS and MTq-PCR.

\begin{tabular}{cccccc}
\hline Method & Comparison & \multicolumn{2}{c}{ Adonis $^{\mathbf{a}}$} & \multicolumn{2}{c}{ Anosim $^{\mathbf{a}}$} \\
\hline & & $\mathbf{R}^{\mathbf{2}}$ & $\boldsymbol{p}$ Value & $\mathbf{R}$ & $p$ Value \\
\hline MTq-PCR & Healthy vs. UC & 0.745 & $<0.001$ & 0.933 & 0.001 \\
NGS & Healthy vs. UC & 0.804 & $<0.001$ & 0.984 & 0.001 \\
\hline
\end{tabular}

\section{Discussion}

Ulcerative colitis (UC) is a chronic inflammatory bowel disease (IBD) that adversely affects the quality of a patient's life $[27,28]$. Recent reports have indicated that UC is characterized by a low diversity of intestinal bacterial flora $[29,30]$. The relative composition of the three major gut flora phyla (Bacteroidetes, Firmicutes, and Proteobacteria) could be a potential diagnostic tool for UC [9,21]. 
Fecal microbiota transplantation (FMT) is a novel therapy for UC that restores the composition and function of the bacterial flora in the gut [31,32]. For FMT to be successful, it is crucial to characterize the presence and absence of individual taxa in the samples of potential fecal donors and UC patients [33]. For decades, next-generation sequencing (NGS) has been used to analyze gut microbiota [34]. Despite the popularity of NGS, the cost and time constraints make it difficult for emergency services to employ it [33-35]. In addition, and of concern because the number of UC patients is rapidly increasing [36], it is more costly to monitor the clinical efficacy of FMT (i.e., analyzing the gut microbiota) of numerous people with NGS. A rapid, sensitive, and cost-effective method is required [20].

TaqMan qPCR is a powerful tool used in the microbial diagnosis of samples from different sources [19]. Hence, in this study, we developed a multiplex TaqMan qPCR (MTq-PCR) with three novel phylum-specific TaqMan probes to determine the proportions of the three dominant phyla in the gut microbiota of UC patients $(n=6)$ and healthy subjects $(n=6)$, namely Bacteroidetes, Firmicutes, and Proteobacteria. The effectiveness of our MTq-PCR assay in profiling the three phyla was compared with NGS.

The phylum-specific probes and primers were designed to target 16S rRNA and were evaluated in silico using the SILVA database [37]. Our probes were comparatively more sensitive than the previously reported probes in discriminating non-target phyla, and they were more specific to gut microbiota [24-26]. This indicates that our probes detected more gut microbiota than the previously reported probes. Our probes exhibited low cross-hybridization to non-target phyla. In addition, phylum-specific primers from previous reports were designed for a singleplex PCR and were less sensitive at discriminating non-target taxa $[17,24]$. In our conventional PCR assay, none of the designed primers and probes interfered with each other during the multiplex PCR reaction, and the reaction was confirmed to be reproducible and sensitive. The multiplex assay showed that the probes were specific, and it was possible to discriminate the three phyla in a single tube. Similar studies reported that real-time PCR assays based on TaqMan hydrolysis probes were specific, sensitive, and rapid compared to conventional PCR $[20,37,38]$. It is important to note that our qPCR assay was based on the proportion of amplicons; therefore, it cannot directly measure the number of individual cells. This is due to the fact that multiple $16 \mathrm{~S}$ rRNA copies are found in different bacteria [39]. In connection with multiple copies of $16 \mathrm{~S}$ rRNA, previous studies similarly reported a biased determination of microbial composition using an NGS approach [40]. To the best of our knowledge, this is the first study to profile human gut microbiota with three different phylum-specific TaqMan probes in a single assay without cross-reactivity.

In our study, both NGS and multiplex PCR methods showed a similar relative abundance between the three major phyla in fecal samples of either UC patients or healthy subjects. Both profiling methods also confirmed that the proportion of Bacteroidetes was greatly reduced in all fecal samples of UC patients when compared to healthy subjects. On the other hand, both methods proved that the relative abundance of Proteobacteria was very high in UC patients when compared to healthy subjects. The results of our study were consistent with a previous report showing that Proteobacteria are integral in the formation of $\mathrm{UC}$, and the load of this phylum in the gut could be an effective diagnostic criterion for UC [21]. Bacteroidetes and Firmicutes are the two dominant phyla in healthy gut microbiota, representing about $90 \%$ of the bacterial population $[13,40,41]$. However, dysbiosis (a microbial imbalance) leads to ulcerative colitis (UC) [3,9]. In our study, both NGS and MTq-PCR showed similar results in determining dysbiosis. PCA and an Anosim and Adonis analysis revealed that NGS and MTq-PCR were not significantly different $(p>0.05)$ in profiling the abundance of the three phyla from fecal samples of either healthy volunteers or UC patients. Both NGS and MTq-PCR indicated significant differences $(p<0.001)$ in the phylum composition between healthy and UC patients. This indicates that our MTq-PCR assay could be used as an alternative method in profiling the three dominant phyla of the gut microbiota since the assay is less costly, rapid, and more accessible than NGS. NGS techniques are costly, time-consuming, and complex for routine applications in resource-limited health care organizations with limited genomic facilities and trained personnel [42]. 
In summary, we developed a rapid, sensitive, and cost-effective MTq-PCR assay that reliably profiled the three major phyla in fecal samples of healthy subjects and UC patients: Bacteroidetes, Firmicutes, and Proteobacteria. The results revealed that the proportion of the three phyla in either healthy subjects or UC patients were similar among NGS and MTq-PCR. This suggests that our assay could be a practical microbiota profiling alternative that can characterize the three phyla involved with gut dysbiosis in UC patients during emergency cases of pre-FMT, and it could also help monitor the clinical efficacy and safety of FMT in UC patients at a low cost.

\section{Materials and Methods}

\subsection{Bacterial Strains}

Three bacterial strains (B. fragilis GUT-04, C. butyricum TO-A, and S. sonnei KCCM41282) representing the three dominant gut flora phyla (Bacteroidetes, Firmicutes, and Proteobacteria, respectively) were obtained from Kyungpook National University culture collections. The strains were grown in fastidious anaerobe broth medium (FAB: peptone, $23 \mathrm{~g}$; soluble starch, $1 \mathrm{~g}$; sodium bicarbonate, $0.4 \mathrm{~g}$; sodium chloride, $5 \mathrm{~g}$; glucose, $1 \mathrm{~g}$; sodium pyruvate, $1 \mathrm{~g}$; L-arginine, $1 \mathrm{~g}$; L-cysteine HCL $0.5 \mathrm{~g}$; sodium pyrophosphate, $0.25 \mathrm{~g}$; sodium succinate, $0.5 \mathrm{~g}$; Hemin, $0.01 \mathrm{~g}$; and Vitamin K, $0.001 \mathrm{~g}$; Seoul, South Korea) at $37^{\circ} \mathrm{C}$ for $48 \mathrm{~h}$.

\subsection{Fecal Sample Collection}

Fresh fecal samples from healthy volunteers $(n=10)$ were collected at Kyungpook National University (KNU), Daegu, South Korea. In addition, fecal samples from six UC patients $(n=6)$ who had been diagnosed with dysbiosis at Yonsei University, Seoul, South Korea were collected. Collection of human fecal samples was performed with the approval of the Institutional Review Board (IRB) at Yonsei University and Kyungpook National University (permit numbers: YSU4-2018-0438 and KNU-2019-0129, respectively). All fecal samples were collected in Transwab ${ }^{\circledR}$ (Medical Wire, UK) following the manufacturer's protocol. Within $24 \mathrm{~h}$, all collected samples were transported to our laboratory in ice packs and stored at $-70{ }^{\circ} \mathrm{C}$ until processing.

\subsection{DNA Extraction}

Genomic DNA from pure cultures of the three bacterial strains was extracted using the Wizard ${ }^{\circledR}$ Genomic Purification Kit (Promega Corporation, USA). DNA from fecal samples was extracted using the QIAamp Powerfecal DNA kit (QIAGEN, Germany) according to the manufacturer's protocol. The DNA concentration was measured using a Qubit ${ }^{\circledR}$ Fluorometer (Thermo Fisher Scientific, Waltham, MA, USA) and stored at $-20^{\circ} \mathrm{C}$ until used.

\subsection{NGS Method}

The V4-V5 variable region of the 16S rRNA gene was PCR amplified using a universal primer pair: forward (515F $5^{\prime}$-GTGCCAGCMGCCGCGG-3') and reverse (907R 5'-CCGTCAATTCMTTTRAGTTT- ${ }^{\prime}$ ). For sequencing, an Ion Torrent PGM adapter and barcode tailored to the primer pair was used. The library preparation reactions consisted of $50 \mu \mathrm{L}$ and were composed of $25 \mu \mathrm{L}$ of EmeraldAmp ${ }^{\circledR}$ Max PCR Master Mix (Takara Korea Biomedical Inc., Seoul, Korea), $1 \mu \mathrm{L}$ of each bacterial primer, $1 \mu \mathrm{L}$ of extracted DNA, and $22 \mu \mathrm{L}$ of ultra-pure water. The first amplification reaction was performed using the following thermocycling program: pre-denaturation at $95^{\circ} \mathrm{C}$ for $3 \mathrm{~min}$, followed by five cycles of $95^{\circ} \mathrm{C}$ for $30 \mathrm{~s}, 57^{\circ} \mathrm{C}$ for $30 \mathrm{~s}$, and $72^{\circ} \mathrm{C}$ for $30 \mathrm{~s}$. The second amplification reaction was 30 cycles of denaturation at $95^{\circ} \mathrm{C}$ for $30 \mathrm{~s}$ and annealing-extension at $72{ }^{\circ} \mathrm{C}$ for $1 \mathrm{~min}$. The final extension was performed at $72{ }^{\circ} \mathrm{C}$ for $5 \mathrm{~min}$ [43].

In this study, NGS was employed, and the quality of the amplified DNA library was assessed using an Agilent 2100 Bioanalyzer High-Sensitivity DNA Assay kit (Agilent Technology, Santa Clara, CA, USA). The pre-amplified DNA library was further diluted to $6 \mathrm{pM}$ to perform emulsion PCR 
with Ion Sphere ${ }^{\mathrm{TM}}$ Particles (ISPs) using the Ion OneTouch System II (Thermo Fisher Scientific Korea Inc., Seoul, Korea) followed by enrichment of template-positive ISPs with Dynabeads ${ }^{\mathrm{TM}}$ MyOne $^{\mathrm{TM}}$ streptavidin C1 beads (Thermo Fisher Scientific, Waltham, MA, USA). Each sample was loaded on an Ion 316 Chip Kit v2 bar-coded chip. Sequencing was performed on the Ion Torrent PGM for 1200 flows with an Ion PGM ${ }^{\mathrm{TM}}$ Hi Q Sequencing Kit (Thermo Fisher Scientific Korea Inc., Seoul, Korea). The Torrent Suite ${ }^{\mathrm{TM}}$ and Ion Torrent PGM-specific pipeline software was employed to generate sequence reads, trim adapter sequences, filter, and remove low-quality signal-profile reads. Quality filtering of generated sequences and taxonomic classification were performed using QIIME software based on the Greengenes database [44].

\subsection{TaqMan Probe and Primer Design}

Five bacterial species from the three dominant phyla in the human gut (Bacteroidetes, Firmicutes, and Proteobacteria) were used for probe design (Table 9.). Complete $16 \mathrm{~S}$ rRNA gene sequences from 15 strains representing the three phyla were downloaded from the National Center for Biotechnology Information (NCBI) GeneBank database. Multiple sequence alignments of the 16S rRNA gene sequences from the 15 strains were performed using CLC Main Workbench 8.1 software (Qiagen, Hilden, Germany). Following alignment, a primer pair targeting the $16 \mathrm{~S}$ rRNA of Eubacteria was designed from a conserved region of all strains, and phylum-specific TaqMan probes were selected from the consensus sequence of each phylum. All probes were designed to be within the binding site of the eubacteria primer pair. A universal probe for eubacteria that targets the opposite strand of the conserved region of the three phyla was used [22]. The specificity of the primer pair and TaqMan probes were confirmed by in silico analysis using the SILVA database [37].

Table 9. Bacterial strains of different phyla used to design phylum-specific primer-probe sets.

\begin{tabular}{ccc}
\hline Target Phylum & Strain & Accession Number \\
\hline Bacteroidetes & Bacteroides fragilis ATCC 25285 & KP326374 \\
\hline & Bacteroides thetaiotaomicron JCM 5827 & NR_112944 \\
\hline Bacteroides coprophilus CB42 (T) & AB260026 \\
\hline Prevotella copri DSM 18205 & NR_040877 \\
\hline Prevotella brevis GA33 & NR_041954 \\
\hline Ruminococcus gnavus ATCC 29149 & NR_036800 \\
\hline Clostridium difficle ATCC 9689 (T) & NR_112172 \\
\hline Faecalibacterium prausnitzii ATCC 27768 (T) & AJ413954 \\
\hline Enterococcus faecium IMAU20967 & MK369883 \\
\hline Roseburia hominis A2-183 (T) & AJ270482 \\
\hline Shigella sonnei CECT 4887 & FR870445 \\
\hline & Enterobacter cloacae ATCC 13047 (T) & NR_102794 \\
\hline Enterobacter aerogenes ATCC 13048 (T) & KF516237 \\
\hline Escherichia coli JCM 1649 & AB242910 \\
\hline & Pseudomonas aeruginosa UTIRB3 & MH910498
\end{tabular}

\subsection{Conventional PCR Assay}

Before conducting the multiplex TaqMan qPCR (mTqPCR) assay, conventional PCR assays were performed to determine the specificity of the designed primer/probe sets using a Mastercycler Nexus PCR machine (Eppendorf, Hamburg, Germany). The primer pairs and TaqMan probes were purchased from Integrated DNA Technologies (IDT, Coralvile, IA, USA). Conventional PCR reactions were $50 \mu \mathrm{L}$ and were composed of $1 \mu \mathrm{L}$ of each forward primer (Eub268), $1 \mu \mathrm{L}$ of universal primer (1492R), $25 \mu \mathrm{L}$ 
of EmeraldAmp Max PCR Master Mix (Takara, Japan), and $1 \mu \mathrm{L}$ of genomic DNA from one of the three strains from the three phyla. Sterilized ultra-pure water was used to make up a total reaction volume of $50 \mu \mathrm{L}$. Genomic DNA from the three strains (B. fragilis GUT-04, C. butyricum TO-A, and S. sonnei KCCM 41282) was added separately. After confirming the specificity of each primer and probe set for their respective phyla, the sets were further evaluated by introducing a mock community (i.e., a mixture of the template DNA from the above-mentioned strains in equal proportions) into the MTq-PCR reaction. All PCR assays were performed with the following thermocycling program: $95^{\circ} \mathrm{C}$ for $5 \mathrm{~min} ; 35$ cycles of $95^{\circ} \mathrm{C}$ for $30 \mathrm{~s}, 60^{\circ} \mathrm{C}$ for $30 \mathrm{~s}$, and $72{ }^{\circ} \mathrm{C}$ for $30 \mathrm{~s}$; and a final extension at $72{ }^{\circ} \mathrm{C}$ for $5 \mathrm{~min}$. PCR mixtures using universal primers (both forward and reverse) served as positive controls. PCR mixtures without a template DNA served as negative controls.

\subsection{Multiplex TaqMan $q P C R$}

In order to quantify the relative proportions of the three phyla (Bacteroidetes, Firmicutes, and Proteobacteria) in fecal samples from UC patients and healthy subjects, a multiplex, real-time TaqMan qPCR was carried out using a CFX96 (Bio-Rad, Hercules, USA). All qPCR reactions for both healthy and UC samples were performed in duplicate, and the average cycle threshold $(\mathrm{Ct})$ values were calculated. Each qPCR reaction was $20 \mu \mathrm{L}$ and was comprised of $10 \mu \mathrm{L}$ of $2 \mathrm{X}$ qPCR master mix (MGmed, Seoul, Korea), $1 \mu \mathrm{L}$ of each primer, $0.5 \mu \mathrm{L}$ of each probe, $1 \mu \mathrm{L}$ of sample DNA, and sterilized ultra-pure water to make up a total reaction volume of $20 \mu \mathrm{L}$. Based on the conventional results, simultaneous quantification of the three phyla was performed with three TaqMan probes labeled with three distinct fluorophores. Bacteriodetes, Firmicutes, Proteobacteria, and Eubacteria probes were labeled with 6-FAM/BHQ1, HEX/BHQ1, Cy5/BHQ2, and TEX615/BHQ2, respectively (Figure 6). All qPCR reactions were performed using the following thermocycling program: initial denaturation at $95^{\circ} \mathrm{C}$ for $5 \mathrm{~min}$ and 40 cycles of $30 \mathrm{~s}$ at $95^{\circ} \mathrm{C}, 30 \mathrm{~s}$ at $60^{\circ} \mathrm{C}$, and $30 \mathrm{~s}$ at $72{ }^{\circ} \mathrm{C}$. The relative proportions of Bacteroidetes, Firmicutes, and Proteobacteria were calculated as follows. The $\mathrm{Ct}$ values of each phylum were normalized by subtracting their respective $\mathrm{Ct}$ values from 40 , and the resulting $\mathrm{Ct}$ values were summed to give a total $\mathrm{Ct}$ value. The relative abundance of each phylum was calculated by dividing their respective normalized $\mathrm{Ct}$ value with the total $\mathrm{Ct}$ value, and was expressed as a percentage by multiplying the resulting ratio by 100 .

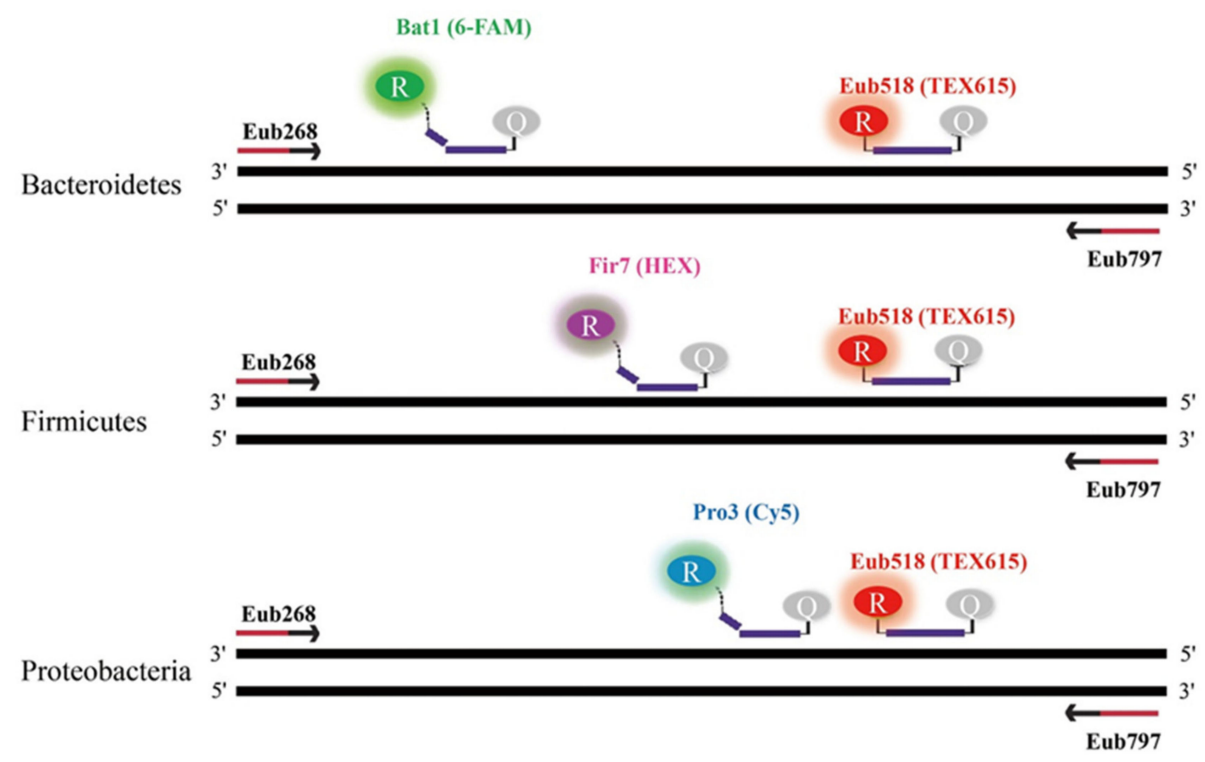

Figure 6. Schematic view of the multiplex TaqMan qPCR for simultaneous detection of the three dominant phyla in gut microflora (Bacteroidetes, Firmicutes, and Proteobacteria) in one PCR reaction. 


\subsection{Statistical Analysis}

A comparison of the relative abundances from NGS and TaqMan qPCR assays was conducted using Calypso version 8.84 [45]. The statistical similarity between NGS and TaqMan qPCR was performed using the analysis of similarities (ANOSIM) test. A dissimilarity analysis was performed using permutational manova (PERMANOVA, Adonis function) [46]. The similarity and dissimilarity analyses were computed based on Bray-Curtis distance.

Author Contributions: Conceptualization, Y.J.J., S.B.T. and J.-H.S.; methodology, Y.J.J. and H.Q.P.; software, H.Q.P.; validation, Y.J.J., S.B.T., Y.J., and J.C.I.; formal analysis, Y.J.J. and J.-H.S.; investigation, Y.J.J., S.B.T. and J.-H.S.; resources, S.P., Y.K., S.K., and H.K.; data curation, Y.J.J., S.C. and G.-U.K.; writing (original draft preparation), Y.J.J., H.Q.P. and S.B.T.; writing (review and editing), S.B.T., and J.-H.S.; visualization, Y.J.J., S.B.T. and J.-H.S.; supervision, H.K. and J.-H.S.; project administration, Y.J.J. and J.-H.S.; and funding acquisition, H.K. and J.-H.S. All authors have read and agreed to the published version of the manuscript.

Funding: This research was financially supported by the Strategic Initiative for Microbiomes in Agriculture and Food (Grant number 918010-4), Ministry of Agriculture, Food and Rural Affairs, Korea. Also, the Bio \& Medical Technology Development Program (2018M3A9H3025030), National Research Foundation funded by the Ministry of Science and ICT, Korea.

Conflicts of Interest: The authors declare no conflicts of interest. The funders had no role in the design of the study; in the collection, analyses, or interpretation of data; in the writing of the manuscript, or in the decision to publish the results.

\section{Abbreviations}

$\begin{array}{ll}\text { ANOSIM } & \text { Analysis of similarities } \\ \text { MTq-PCR } & \text { Multiplex TaqMan quantitative polymerase chain reaction } \\ \text { IBD } & \text { Inflammatory bowel disease } \\ \text { UC } & \text { ulcerative colitis } \\ \text { FMT } & \text { Fecal microbiota transplantation } \\ \text { NGS } & \text { Next generation sequencing } \\ \text { 6-FAM } & \text { 6-carboxylfluorescein } \\ \text { HEX } & \text { Hexachloro-fluorescein } \\ \text { Cy5 } & \text { Cyanine dye } \\ \text { TEX615 } & \text { Texas red fluorophore } \\ \text { BHQ-1 } & \text { Black hole quencher-1 } \\ \text { BHQ-2 } & \text { Black hole quencher-1 }\end{array}$

\section{References}

1. Hooper, L.V.; MacPherson, A.J. Immune adaptations that maintain homeostasis with the intestinal microbiota. Nat. Rev. Immunol. 2010, 10, 159-169. [CrossRef] [PubMed]

2. Icaza-Chávez, M.E. Gut microbiota in health and disease. Rev. Gastroenterol. México Engl. Ed. 2013, 78, 240-248.

3. Wang, B.; Yao, M.; Lv, L.; Ling, Z.; Li, L. The Human Microbiota in Health and Disease. Engineering 2017, 3 , 71-82. [CrossRef]

4. Shivashankar, R.; Tremaine, W.J.; Harmsen, W.S.; Loftus, E.V. Incidence and Prevalence of Crohn's Disease and Ulcerative Colitis in Olmsted County, Minnesota From 1970 Through 2010. Clin. Gastroenterol. Hepatol. 2017, 15, 857-863. [CrossRef] [PubMed]

5. Marlicz, W. World digestive health day—WDHD Poland 2017 inflammatory bowel diseases—Challenges and hopes. Prz. Gastroenterol. 2018, 13, 82-84. [CrossRef] [PubMed]

6. Shi, Y.; Dong, Y.; Huang, W.; Zhu, D.; Mao, H.; Su, P. Fecal microbiota transplantation for ulcerative colitis: A systematic review and meta-analysis. PLoS ONE 2016, 11, e0157259. [CrossRef] [PubMed]

7. Uygun, A.; Ozturk, K.; Demirci, H.; Oger, C.; Avci, I.Y.; Turker, T.; Gulsen, M. Fecal microbiota transplantation is a rescue treatment modality for refractory ulcerative colitis. Medicine 2017, 96, e6479. [CrossRef] 
8. Wang, H.G.; Liu, S.P.; Ma, T.H.; Yan, W.; Zhou, J.F.; Shi, Y.T.; Shen, P.; Yang, X.Z.; Wu, S.N. Fecal microbiota transplantation treatment for refractory ulcerative colitis with allergy to 5-aminosalicylic acid: A case report. Medicine 2018, 97, e0675. [CrossRef]

9. Fuentes, S.; Rossen, N.G.; Van Der Spek, M.J.; Hartman, J.H.A.; Huuskonen, L.; Korpela, K.; Salojärvi, J.; Aalvink, S.; De Vos, W.M.; D'Haens, G.R.; et al. Microbial shifts and signatures of long-term remission in ulcerative colitis after faecal microbiota transplantation. ISME J. 2017, 11, 1877-1889. [CrossRef]

10. Fairhurst, N.G.; Travis, S.P.L. Why is it so difficult to evaluate faecal microbiota transplantation as a treatment for ulcerative colitis? Intest. Res. 2018, 16, 209. [CrossRef]

11. Basso, P.J.; Saraiva Câmara, N.O.; Sales-Campos, H. Microbial-based therapies in the treatment of inflammatory bowel disease-An overview of human studies. Front. Pharmacol. 2019, 9, 1571. [CrossRef] [PubMed]

12. Flannigan, K.L.; Rajbar, T.; Moffat, A.; McKenzie, L.S.; Dicke, F.; Rioux, K.; Workentine, M.L.; Louie, T.J.; Hirota, S.A.; Greenway, S.C. Changes in composition of the gut bacterial microbiome after fecal microbiota transplantation for recurrent Clostridium difficile infection in a pediatric heart transplant patient. Front. Cardiovasc. Med. 2017, 4, 17. [CrossRef] [PubMed]

13. Wang, Z.; Xu, C.M.; Liu, Y.X.; Wang, X.Q.; Zhang, L.; Li, M.; Zhu, S.W.; Xie, Z.J.; Wang, P.H.; Duan, L.P.; et al. Characteristic dysbiosis of gut microbiota of Chinese patients with diarrhea-predominant irritable bowel syndrome by an insight into the pan-microbiome. Chin. Med. J. 2019, 132, 889. [CrossRef] [PubMed]

14. D'Argenio, V.; Salvatore, F. The role of the gut microbiome in the healthy adult status. Clin. Chim. Acta. 2015, 451, 97-102. [CrossRef] [PubMed]

15. Okamoto, K.; Watanabe, T.; Komeda, Y.; Okamoto, A.; Minaga, K.; Kamata, K.; Yamao, K.; Takenaka, M.; Hagiwara, S.; Sakurai, T.; et al. Dysbiosis-associated polyposis of the colon-cap polyposis. Front. Immunol. 2018, 9, 918. [CrossRef]

16. Sasaki, K.; Inoue, J.; Sasaki, D.; Hoshi, N.; Shirai, T.; Fukuda, I.; Azuma, T.; Kondo, A.; Osawa, R. Construction of a Model Culture System of Human Colonic Microbiota to Detect Decreased Lachnospiraceae Abundance and Butyrogenesis in the Feces of Ulcerative Colitis Patients. Biotechnol. J. 2019, 14, 1800555. [CrossRef]

17. Haakensen, M.; Dobson, C.M.; Deneer, H.; Ziola, B. Real-time PCR detection of bacteria belonging to the Firmicutes Phylum. Int. J. Food Microbiol. 2008, 125, 236-241. [CrossRef]

18. Linck, H.; Krüger, E.; Reineke, A. A multiplex TaqMan qPCR assay for sensitive and rapid detection of phytoplasmas infecting Rubus species. PLoS ONE 2017, 12, e0177808. [CrossRef]

19. Nagy, A.; Vitásková, E.; Černíková, L.; Křivda, V.; Jiřincová, H.; Sedlák, K.; Horníčková, J.; Havlíčková, M. Evaluation of TaqMan qPCR system integrating two identically labelled hydrolysis probes in single assay. Sci. Rep. 2017, 7, 41392. [CrossRef]

20. Xu, X.; Yang, F.; Zhang, Q.; Xu, Y.; Huang, J.; Fu, M.; Zhang, W. Development of a multiplex TaqMan qPCR assay for simultaneous detection and differentiation of four DNA and RNA viruses from clinical samples of sheep and goats. J. Virol. Methods 2019, 266, 58-64. [CrossRef]

21. Shin, N.R.; Whon, T.W.; Bae, J.W. Proteobacteria: Microbial signature of dysbiosis in gut microbiota. Trends Biotechnol. 2015, 33, 496-503. [CrossRef] [PubMed]

22. Muyzer, G.; De Waal, E.C.; Uitterlinden, A.G. Profiling of complex microbial populations by denaturing gradient gel electrophoresis analysis of polymerase chain reaction-amplified genes coding for 16S rRNA. Appl. Environ. Microbiol. 1993, 59, 695-700. [CrossRef] [PubMed]

23. Nadkarni, M.A.; Martin, F.E.; Jacques, N.A.; Hunter, N. Determination of bacterial load by real-time PCR using a broad-range (universal) probe and primers set. Microbiology 2002, 148, 257-266. [CrossRef] [PubMed]

24. Pfeiffer, S.; Pastar, M.; Mitter, B.; Lippert, K.; Hackl, E.; Lojan, P.; Oswald, A.; Sessitsch, A. Improved group-specific primers based on the full SILVA 16S rRNA gene reference database. Environ. Microbiol. 2014, 16, 2389-2407. [CrossRef] [PubMed]

25. Mühling, M.; Woolven-Allen, J.; Murrell, J.C.; Joint, I. Improved group-specific PCR primers for denaturing gradient gel electrophoresis analysis of the genetic diversity of complex microbial communities. ISME J. 2008, 2, 379-392. [CrossRef]

26. Bacchetti De Gregoris, T.; Aldred, N.; Clare, A.S.; Burgess, J.G. Improvement of phylum- and class-specific primers for real-time PCR quantification of bacterial taxa. J. Microbiol. Methods 2011, 86, 351-356. [CrossRef] 
27. Yang, Y.W.; Chen, M.K.; Yang, B.Y.; Huang, X.J.; Zhang, X.R.; He, L.Q.; Zhang, J.; Hua, Z.C. Use of $16 S$ rRNA gene-targeted group-specific primers for real-time PCR analysis of predominant bacteria in mouse feces. Appl. Environ. Microbiol. 2015, 81, 6749-6756. [CrossRef]

28. Kozłowska, K.A.; Ba sczyk, G.; Krokowicz, P. Quality of life in patients with ulcerative colitis treated surgically. Prz. Gastroenterol. 2014, 9, 220. [CrossRef]

29. Borody, T.J.; Clancy, A. Fecal microbiota transplantation for ulcerative colitis-where to from here? Transl. Gastroenterol. Hepatol. 2019, 4. [CrossRef]

30. Walujkar, S.A.; Dhotre, D.P.; Marathe, N.P.; Lawate, P.S.; Bharadwaj, R.S.; Shouche, Y.S. Characterization of bacterial community shift in human ulcerative colitis patients revealed by Illumina based 16S rRNA gene amplicon sequencing. Gut Pathog. 2014, 6, 22. [CrossRef]

31. Vrieze, A.; de Groot, P.F.; Kootte, R.S.; Knaapen, M.; Van Nood, E.; Nieuwdorp, M. Fecal transplant: A safe and sustainable clinical therapy for restoring intestinal microbial balance in human disease? Best Pract. Res. Clin. Gastroenterol. 2013, 27, 127-137. [CrossRef] [PubMed]

32. Shen, Z.H.; Zhu, C.X.; Quan, Y.S.; Yang, Z.Y.; Wu, S.; Luo, W.W.; Tan, B.; Wang, X.Y. Relationship between intestinal microbiota and ulcerative colitis: Mechanisms and clinical application of probiotics and fecal microbiota transplantation. World J. Gastroenterol. 2018, 24, 5. [CrossRef] [PubMed]

33. Duvallet, C.; Zellmer, C.; Panchal, P.; Budree, S.; Osman, M.; Alm, E.J. Framework for rational donor selection in fecal microbiota transplant clinical trials. PLoS ONE 2019, 14, e222881. [CrossRef]

34. Millat, G.; Chanavat, V.; Rousson, R. Evaluation of a new NGS method based on a custom AmpliSeq library and Ion Torrent PGM sequencing for the fast detection of genetic variations in cardiomyopathies. Clin. Chim. Acta 2014, 433, 266-271. [CrossRef] [PubMed]

35. Reiman, A.; Kikuchi, H.; Scocchia, D.; Smith, P.; Tsang, Y.W.; Snead, D.; Cree, I.A. Validation of an NGS mutation detection panel for melanoma. BMC Cancer 2017, 17, 150. [CrossRef]

36. Ananthakrishnan, A.N.; McGinley, E.L.; Saeian, K.; Binion, D.G. Temporal trends in disease outcomes related to clostridium difficile infection in patients with inflammatory bowel disease. Inflamm. Bowel Dis. 2011, 17, 976-983. [CrossRef] [PubMed]

37. Quast, C.; Pruesse, E.; Yilmaz, P.; Gerken, J.; Schweer, T.; Yarza, P.; Peplies, J.; Glöckner, F.O. The SILVA ribosomal RNA gene database project: Improved data processing and web-based tools. Nucleic Acids Res. 2013, 41, D590-D596. [CrossRef]

38. Venkatesan, G.; Bhanuprakash, V.; Balamurugan, V.; Prabhu, M.; Pandey, A.B. TaqMan hydrolysis probe based real time PCR for detection and quantitation of camelpox virus in skin scabs. J. Virol. Methods 2012, 181, 192-196. [CrossRef]

39. Ibal, J.C.; Pham, H.Q.; Park, C.E.; Shin, J.H. Information about variations in multiple copies of bacterial $16 \mathrm{~S}$ rRNA genes may aid in species identification. PLoS ONE 2019, 14, e0212090. [CrossRef]

40. Boers, S.A.; Jansen, R.; Hays, J.P. Understanding and overcoming the pitfalls and biases of next-generation sequencing (NGS) methods for use in the routine clinical microbiological diagnostic laboratory. Eur. J. Clin. Microbiol. Infect. Dis. 2019, 38, 1059-1070. [CrossRef]

41. Fujio-Vejar, S.; Vasquez, Y.; Morales, P.; Magne, F.; Vera-Wolf, P.; Ugalde, J.A.; Navarrete, P.; Gotteland, M. The gut microbiota of healthy Chilean subjects reveals a high abundance of the phylum Verrucomicrobia. Front. Microbiol. 2017, 8, 1221. [CrossRef] [PubMed]

42. Deurenberg, R.H.; Bathoorn, E.; Chlebowicz, M.A.; Couto, N.; Ferdous, M.; García-Cobos, S.; Kooistra-Smid, A.M.D.; Raangs, E.C.; Rosema, S.; Veloo, A.C.M.; et al. Application of next generation sequencing in clinical microbiology and infection prevention. J. Biotechnol. 2017, 243, 16-24. [CrossRef] [PubMed]

43. Caporaso, J.G.; Kuczynski, J.; Stombaugh, J.; Bittinger, K.; Bushman, F.D.; Costello, E.K.; Fierer, N.; Pẽa, A.G.; Goodrich, J.K.; Gordon, J.I.; et al. QIIME allows analysis of high-throughput community sequencing data. Nat. Methods 2010, 7, 335. [CrossRef] [PubMed]

44. Rutgeerts, P.; Sandborn, W.J.; Feagan, B.G.; Reinisch, W.; Olson, A.; Johanns, J.; Travers, S.; Rachmilewitz, D.; Hanauer, S.B.; Lichtenstein, G.R.; et al. Infliximab for induction and maintenance therapy for ulcerative colitis. N. Engl. J. Med. 2005, 353, 2462-2476. [CrossRef] [PubMed] 
45. Zakrzewski, M.; Proietti, C.; Ellis, J.J.; Hasan, S.; Brion, M.J.; Berger, B.; Krause, L. Calypso: A user-friendly web-server for mining and visualizing microbiome-environment interactions. Bioinformatics 2017, 33, 782-783. [CrossRef] [PubMed]

46. Anderson, M.J. A new method for non-parametric multivariate analysis of variance. Austral Ecol. 2001, 26, 32-46.

(C) (1)

(C) 2020 by the authors. Licensee MDPI, Basel, Switzerland. This article is an open access article distributed under the terms and conditions of the Creative Commons Attribution (CC BY) license (http://creativecommons.org/licenses/by/4.0/). 